\title{
HIDRATO DE GÁS SUBMARINO: NATUREZA, OCORRÊNCIA E PERSPECTIVAS PARA EXPLORAÇÃO NA MARGEM CONTINENTAL BRASILEIRA
}

\author{
Michael B. Clennell \\ Received August 23, 2000 / Accepted September 03, 2001
}

\begin{abstract}
Hidrato de gás, ou clatrato é um sólido cristalino sendo composto de água e gases de peso molecular pequeno. Os hidratos de metano são abundantes em sedimentos submarinhos nas margens continentais. A distribuição dos clatratos pode ser mapeada através de perfilagem sísmica, perfis de poço, e amostragem geoquímica. A quantidade estimada de hidratos de gás submarino no mundo equivale aproximadamente a duas vezes o total de todos os recursos convencionais de óleo e gás. Entretanto, a exploração de hidratos de gás submarino como fonte de energia ainda não é viável em termos técnicos ou econômicos. Deslizamentos de grandes proporções podem ser desencadeados pela dissociação dos hidratos. O gás liberado durante um evento dessa natureza pode entrar na atmosfera, estimulando o efeito estufa. $\mathrm{O}$ talude continental do Brasil mostra em várias localidades assinaturas geofísicas da presença de hidratos de gás, e isto não é incomum, uma vez que as condições geológicas adequadas para a formação deste mineral são encontradas em outras áreas da margem continental. Apesar da existência de recurso como os hidrocarbonetos não-convencionais em águas brasileiras, esses também apresentam um risco desconhecido quanto às operações de exploração e de produção em campos de óleo e gás já em desenvolvimento em águas profundas.
\end{abstract}

Palavras-chave: Hidrato de gás; Margem continental; Geoquímica orgânica; Recursos marinhos.

\begin{abstract}
SUBMARINE GAS HYDRATES: NATURE, OCCURRENCE \& PERSPECTIVES FOR EXPLORATION IN THE BRAZILIAN CONTINENTAL MARGIN - Gas hydrates or clathrates are crystalline compounds of water and gas. Natural clathrates trapping mostly methane, are widespreads in the sediments of the continental margins between water depths of $500-3500 \mathrm{~m}$. Clathrates, are rarely sampled in cores but can be detected indirectly by geochemical tracers and by the response of downhole well logs. The extent of hydrates can be mapped using characteristic reflectors and amplitude anomalies on seismic lines. Estimated on this basis, the quantity of methane in marine clathrates is enormous; equivalent in energy value to about twice conventional hydrocarbon resources worldwide. However, exploitation of marine hydrates as a fuel is not as yet technically or economically viable. Major submarine landslides may be triggered by hydrate dissociation due to sea level fall or sea bottom temperature increase. The released gas may enter the atmosphere, and contribute to greenhouse warming. The Brazilian continental margin shows geophysical indicators of gas hydrates in several localities, and geochemical conditions are favourable in others. This gives the possibility of unconventional hydrocarbon plays, but may also pose a geotechnical risk to drilling and production activity.
\end{abstract}

Key words: Gas hydrates; Continental margin; Organic geochemistry; Marine mineral resources

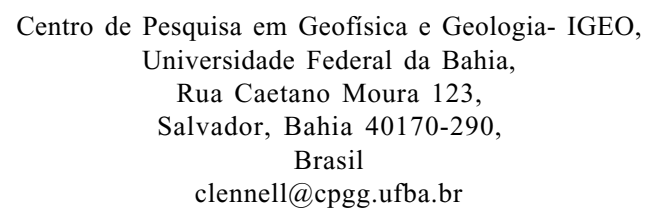




\section{INTRODUÇÃO}

Hidrato de gás ou clatrato, que em latim significa "gaiola", é um composto cristalino no qual as moléculas de água, associadas umas às outras com ligações de hidrogênio, encapsulam moléculas de gás como o metano e dióxido de carbono (Sloan, 1998;
Tabela 1). O tipo de ligação intermolecular é parecido com a estrutura do gelo comum, mas o arranjo geométrico produz cavidades que cercam os gases originalmente em solução na água. Apesar da ausência de ligações químicas entre hospedeiro (água) e hóspede (metano, etc.), a estrutura estável do clatrato permite que o "gelo" não se derreta até temperaturas

\begin{tabular}{lcc}
\hline Propriedade & Gelo & Hidrato de Metano \\
\hline Densidade $\left(\mathrm{kgm}^{-3}\right)$ & 916 & 912 \\
Módalo de incompressibilidade $(\mathrm{Pa})$ & $8.8 \times 10^{9}$ & $5.6 \times 10^{9}$ \\
Módulo de cisalhamento $(\mathrm{Pa})$ & $3.9 \times 10^{9}$ & $2.4 \times 10^{9}$ \\
Constante dielétrica $(-)$ & 94 & 58 \\
Condutividade térmica W m-l $\mathrm{K}^{-1}$ & 2.25 & 0.5 \\
Entalpia de fusão $\left(\mathrm{Jmol}^{-1}\right)$ & 6000 & 55000 \\
\hline
\end{tabular}

Tabela 1 - Propriedades físicas dos hidratos de gás e do gelo comum.

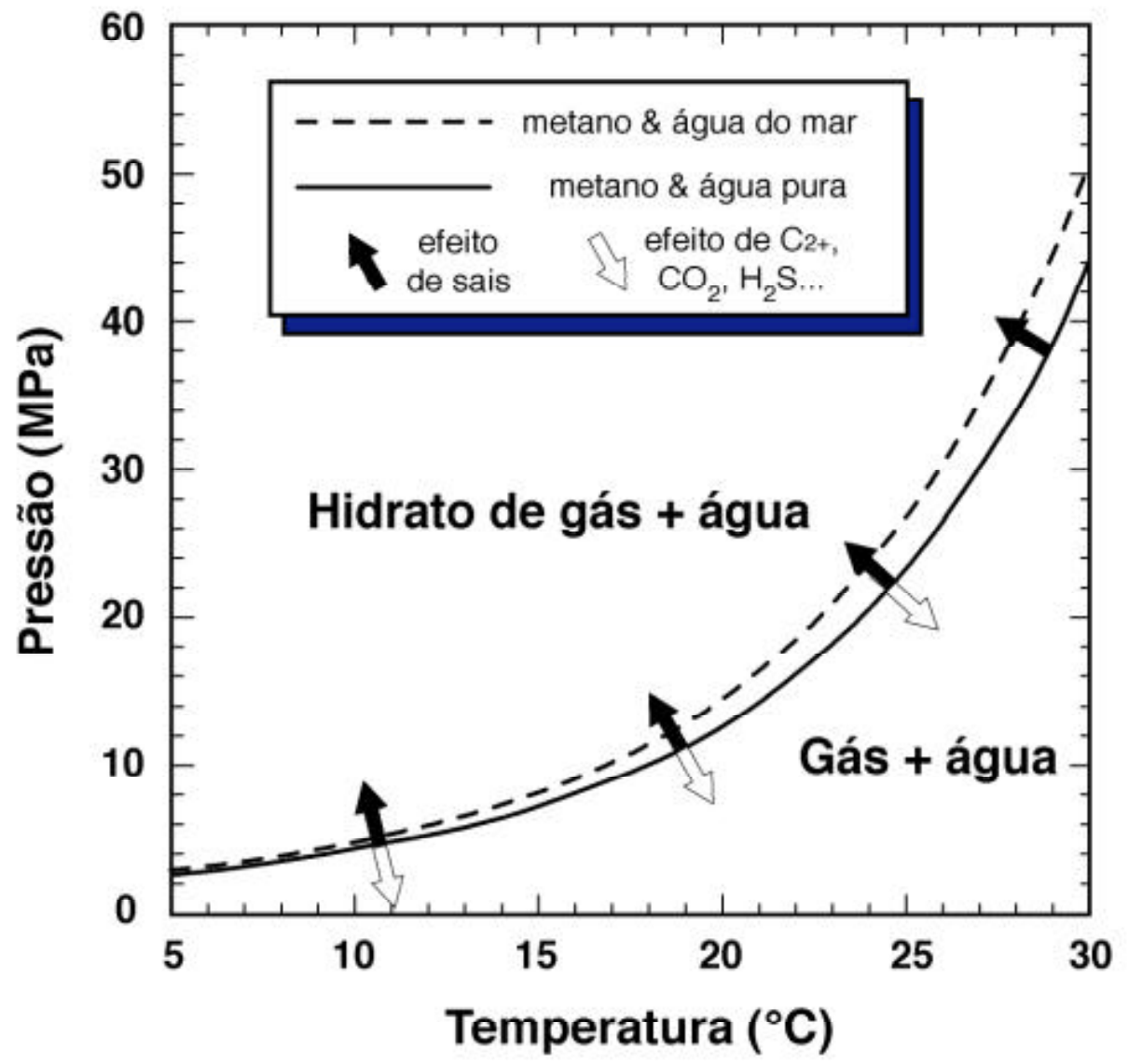

Figura 1 - Diagrama de fase mostrando as condições de pressão e temperatura adequadas pela estabilidade termodinâmica de hidratos de gás. Flechas brancas indicam a influencia de gases como dióxido, que servem para promover a estabilidade do clatrato. Flechas pretas mostram como sais reduzem a estabilidade através de uma queda em atividade química da água. 


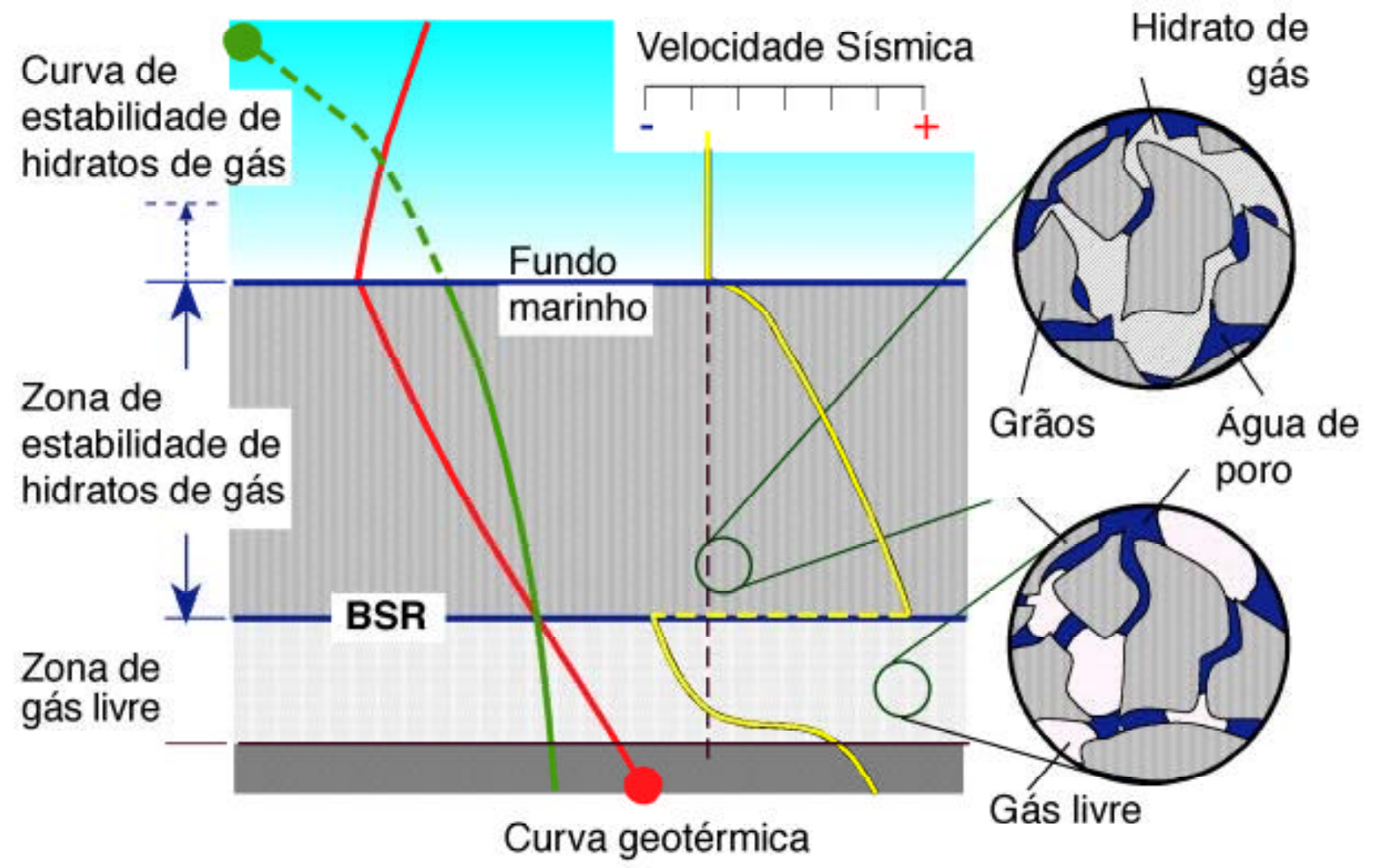

Figura 2 - A zona de estabilidade de hidratos de gás esta limitada pela intersecção da curva de estabilidade do clatrato com a curva de temperatura dentro dos sedimentos marinhos. Embaixo da zona com hidratos de gás existe normalmente uma zona de gás livre liberada pela desassociação do clatrato. A camada de sedimentos com gás dentro do espaço poroso cria uma zona de baixa velocidade sísmica, que produz um refletor paralelo ao fundo marinho que se chama o BSR.

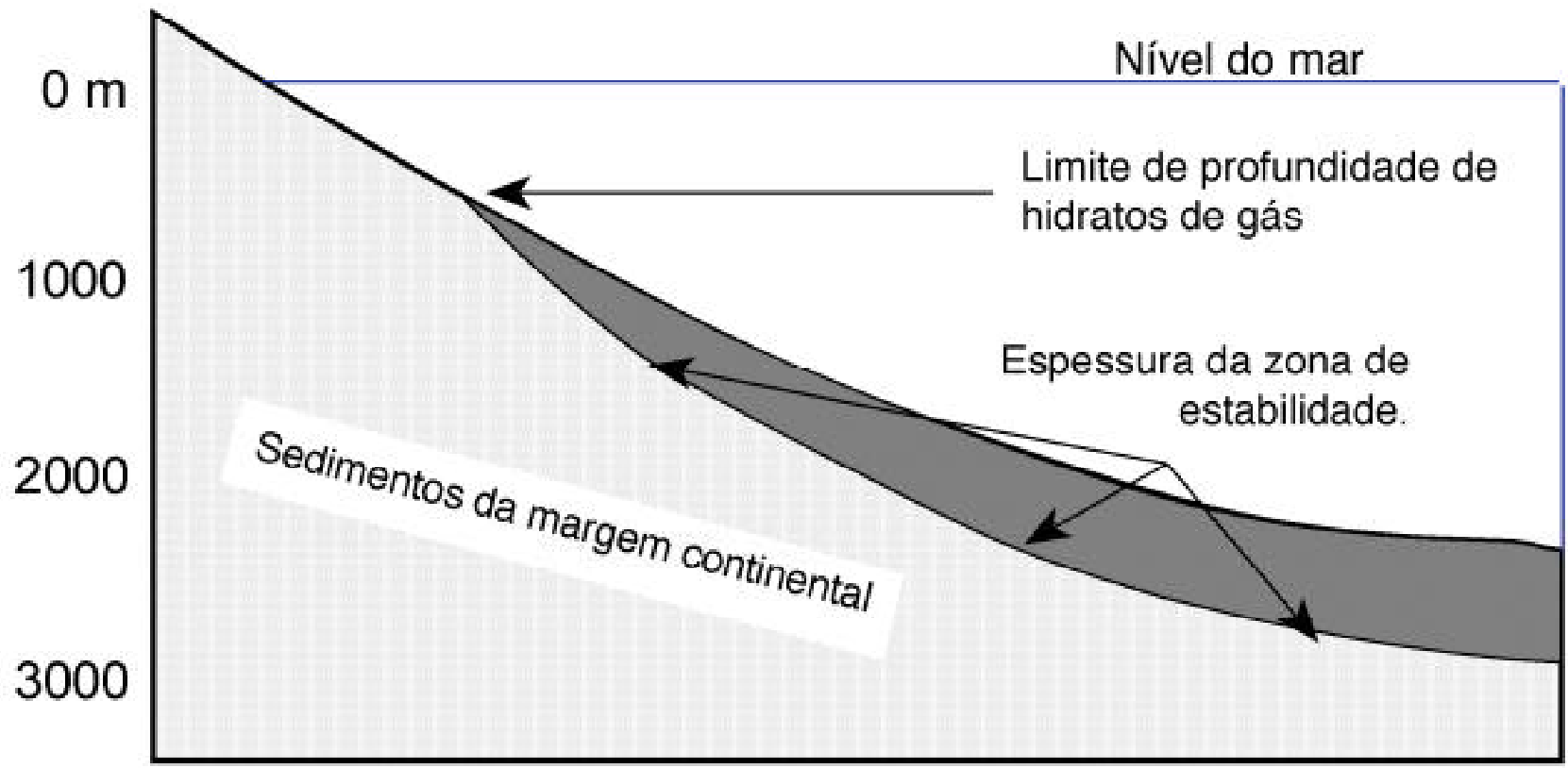

Figura 3 - A espessura da zona de estabilidade de hidratos de gás na margem continental é controlada pela pressão hidrostática e pelo gradiente em temperatura que existe dentro dos sedimentos. Com uma lâmina de água mais profunda, a temperatura de derretimento dos hidratos fica mais alta, e a espessura da camada de sedimentos que pode suportar a ocorrência de hidratos fica mais extensa. O limite de profundidade necessário para encontrar hidratos de gás é aproximadamente $500 \mathrm{~m}$, e depende da temperatura no fundo marinho. A figura mostra os limites de estabilidade: a presença de hidratos depende também do conteúdo de metano nos sedimentos. 
bem acima de $0^{\circ} \mathrm{C}$ desde que a pressão predominante e a concentração de gases sejam suficientemente altas (Fig. 1). Estas condições são encontradas em sedimentos marinhos nas margens continentais onde a lâmina de água é superior a aproximadamente 500 $\mathrm{m}$ (Figs. 2 e 3). A molécula hóspede mais comum em hidratos de gás marinho é o metano $\left(\mathrm{CH}_{4}\right)$; os hidrocarbonetos mais pesados e moléculas como o $\mathrm{CO}_{2}$ e o $\mathrm{H}_{2} \mathrm{~S}$ normalmente ocorrem em proporções menores que $2 \%$ (Kvenvolden, 1993). Enquanto o $\mathrm{CO}_{2}, \mathrm{o}_{2} \mathrm{~S}$ e o $\mathrm{C} 2+$ servem para aumentar a temperatura de dissociação do clatrato (Fig 1), a presença de sais dissolvidos na água reduz a estabilidade deste.

Os clatratos são abundantes nas margens continentais do mundo, formando um reservatório gigantesco, potencialmente móvel e que está integrado no ciclo do carbono. Uma estimativa recente da massa total de carbono em hidratos de gás marinho é de $1.5 \times 10^{16} \mathrm{~kg}$ (Buffett, 2000). Convertida em energia, esta quantidade de metano equivale a duas vezes o total de recursos fósseis já descobertos, sendo uma possibilidade para a exploração no futuro. O metano liberado do derretimento dos hidratos pode provocar mudanças climáticas (Harvey \& Huang, 1995; Haq, 2000). A decomposição de hidratos também pode afetar a estabilidade dos taludes submarinos (Carpenter, 1981), porque o gás liberado gera sobrepressão, e além do mais o sedimento contendo gás é menos coeso que o sedimento cimentado por clatrato (Paull et al., 2000).

\section{FONTES DE METANO}

Os hidratos de gás são formados quando uma quantidade de metano for produzida por bactérias em pequenas profundidades no pacote sedimentar $(<1000 \mathrm{~m})$ e quando houver desestruturação da matéria orgânica em zonas profundas onde temperatura atinge $80-100^{\circ} \mathrm{C}$ (Paull et al., 1994). O principal gás biogênico é o metano $(>99 \% \mathrm{C} 1)$, o qual apresenta teores de $?{ }^{13} \mathrm{C}$ entre $-80 \%$ e $-100 \%$. Já os gases termogênicos mostram teores de ? ${ }^{13} \mathrm{C}$ entre $-30 \%$ e $-40 \%$, e apresentam uma mistura de metano e outros hidrocarbonetos (C2-C5). Enquanto o metano biogênico pode ser produzido na zona de estabilidade dos clatratos, os gases termogênicos migram verticalmente a partir das rochas geradoras até chegar a esta zona.

As bactérias metanogênicas presentes nos sedimentos são capazes de reduzir a metano qualquer dióxido de carbono ou acetato formado como produto secundário de outras atividades biológicas (Wellsbury \& Parkes, 2000). Um dos requisitos para que este metabolismo ocorra é que haja a ausência de espécies redutoras como o sulfato que está presente na água do mar. Desta forma, a metanogênese geralmente inicia à base da zona de redução do sulfato, a alguns metros abaixo do fundo marinho. É necessário que o teor de material orgânico esteja entre $0,5 \%$ e $1,0 \%$ para que os clatratos sejam produzidos em quantidades apreciáveis. Estes teores de matéria orgânica são raros longe dos continentes, o que explica a ausência de hidratos de gás em oceanos abertos. Além do mais, uma taxa de sedimentação alta é um pré-requisito para o soterramento e preservação da matéria orgânica, mantendo-a fora do alcance dos organismos aeróbicos, e das bactérias redutoras de nitrato, ferro e sulfato. Vários trechos da margem continental brasileira atingem os critérios descritos acima para a presença de hidratos de gás biogênicos; ou seja, apresentam teores de matéria orgânica disponível $>0,5$ $\%$, temperatura do fundo marinho $<15^{\circ} \mathrm{C}$ e profundidade $>500 \mathrm{~m}$. Além disso, várias bacias petrolíferas na margem continental brasileira podem acomodar também depósitos de clatratos termogênicos.

\section{FORMAÇÃO E DISTRIBUIÇÃO DE HIDRATOS DE GÁS SUBMARINOS}

O principal fator que controla a estabilidade termodinâmica e a distribuição espacial dos hidratos de gás é a variação em solubilidade de metano dentro da água dos poros. A solubilidade do metano em água é baixa, e varia em função da temperatura e da pressão hidrostática. As características físicas e químicas dos sedimentos também influenciam a forma de crescimento e estabilidade deste mineral. A temperatura aumenta com a profundidade nos sedimentos, e devido a isso, a curva geotérmica eventualmente cruza a linha de estabilidade termodinâmica do hidrato de gás. No caso de ocorrer 
um alto teor de metano no nível onde as linhas se cruzam, serão encontradas bolhas de gás livre abaixo, e cristais de clatrato acima do limite de fase (Figs. $1 \mathrm{e}$ 3). O gás livre e os clatratos representam o excesso de metano acima da solubilidade no sistema. No caso de haver um baixo conteúdo de metano no sistema, não serão encontradas na vizinhança do limite de fase nem clatratos ou gases livres: será encontrado somente metano em solução, em concentração abaixo do nível de saturação. Entretanto, nas condições mais frias encontradas nos níveis mais rasos, se a mesma concentração de metano estiver disponível (sendo transportado por difusão e advecção nos poros), a solubilidade será excedida, levando à precipitação de clatrato. Davie \& Buffett (2001) mostraram que em muitas margens continentais, onde há a ausência de sobre-pressão, o fluxo vertical do metano é pequeno. Desta forma, uma seqüência típica de sedimentos numa margem passiva, com teor de carbono disponível de $1 \%$, pode produzir no máximo $2-7 \%$ de clatrato de metano no espaço poroso. Seriam necessárias condições especiais, como uma grande geração e transporte de metano, para que fossem produzidos hidratos de gás em quantidades econômicas (Hovland et al., 1997; Clennell et al., 2000). Em focos de migração, como em zonas de fraturas e falhas, o gás pode percolar através dos sedimentos até o fundo marinho, criando exudações ou vulcões de lama. Nestas localidades são encontrados veios e nódulos de clatrato em abundância (Ginsburg \& Soloviev, 1997). As exudações sustentam comunidades biossintéticas, incluindo pelo menos uma espécie de artrópode que consome hidratos de gás (Fisher et al., 2000).

Nas margens continentais convergentes, o fluxo adicional de água induzida pela compressão tectônica pode aumentar o transporte de metano termogênico. Na verdade, algumas das maiores jazidas de clatratos de metano têm sido encontradas em margens ativas da América Central, Cascadia, e no Japão.

\section{DETECÇÃO GEOFÍSICA DE HIDRATOS DE GÁS}

A presença de hidratos de gás é freqüentemente inferida devido à aparência em perfis sísmicos de um refletor forte, de amplitude negativa e sub-paralelo ao fundo mar (Shipley et al., 1979; Fig 4), conhecido como o BSR (Bottom Simulating Reflector, em inglês). O BSR marca a profundidade máxima de estabilidade de clatrato de metano (Figs. 2, 3). Perfurações feitas pelo Ocean Drilling Program revelaram uma zona basal de sedimentos com gás livre, de pequena velocidade sísmica superposto por uma zona de sedimentos com velocidade acima do normal devido à cimentação (fraca ou forte) pelos cristais de clatrato (Holbrook et al., 1996). Em alguns lugares, a presença de clatratos revele-se como uma redução nos amplitudes dos refletores sísmicas: a chamada Blanking ou "blindagem" (Lee et al, 1993; Fig. 4). Blanking é provocada por cimentação, que reduz o contraste em impedância sísmica entre camadas de sedimentos de textura diferente.

Métodos modernos de aquisição e processamento de dados sísmicos como inversão completa da onda (Minshull et al., 1994), analise de velocidade de alta resolução (Wood et al. 1994); tomografia em tempo de transito (Tinivella et al., 1998), e análise de AVO (Andreassen et al. 1997; Ecker et al., 2000) permitem a detecção confiável e quantificação parcial das acumulações de hidratos de gás submarinhas. Recentemente sugiram estudos usando sísmica 3-D e 4C (Pecher e Holbrook, 2000). Ondas de cisalhamento (tipo S) que podem ser detectados usando sensores no fundo marinho (OBS) carregam uma dimensão adicional aos dados de velocidade e impedância usando exclusivamente ondas P (Hobro et al., 1998). Especificamente, a onda de cisalhamento e pouco atenuada para gás livre e a velocidade da onda $\mathrm{S}$ é muito mais sensível à rigidez dos sedimentos, que é fortemente influenciada pela cimentação induzida pelos hidratos de gás até em quantidades pequenas (Dvorkin et al., 2000).

A ferramenta principal disponível ao geólogo ou geofísico marinho com interesse em prospecção para hidratos de gás e o levantamento sísmico (Miles, 2000), mas outros métodos de sensoriamento remoto, como sondagens geoelétricas (Edwards et al., 1997) e levantamentos da conformabilidade elástica do fundo marinho (Willoughby et al., 2000) também estão em desenvolvimento. Além disto, é necessário perfurar para alcançar diretamente as camadas contendo hidratos de gás. O uso de sondas de perfilagem elétrica em poços permite a coleta de dados contínuos das 


\section{USGS LINE 92-12-16}
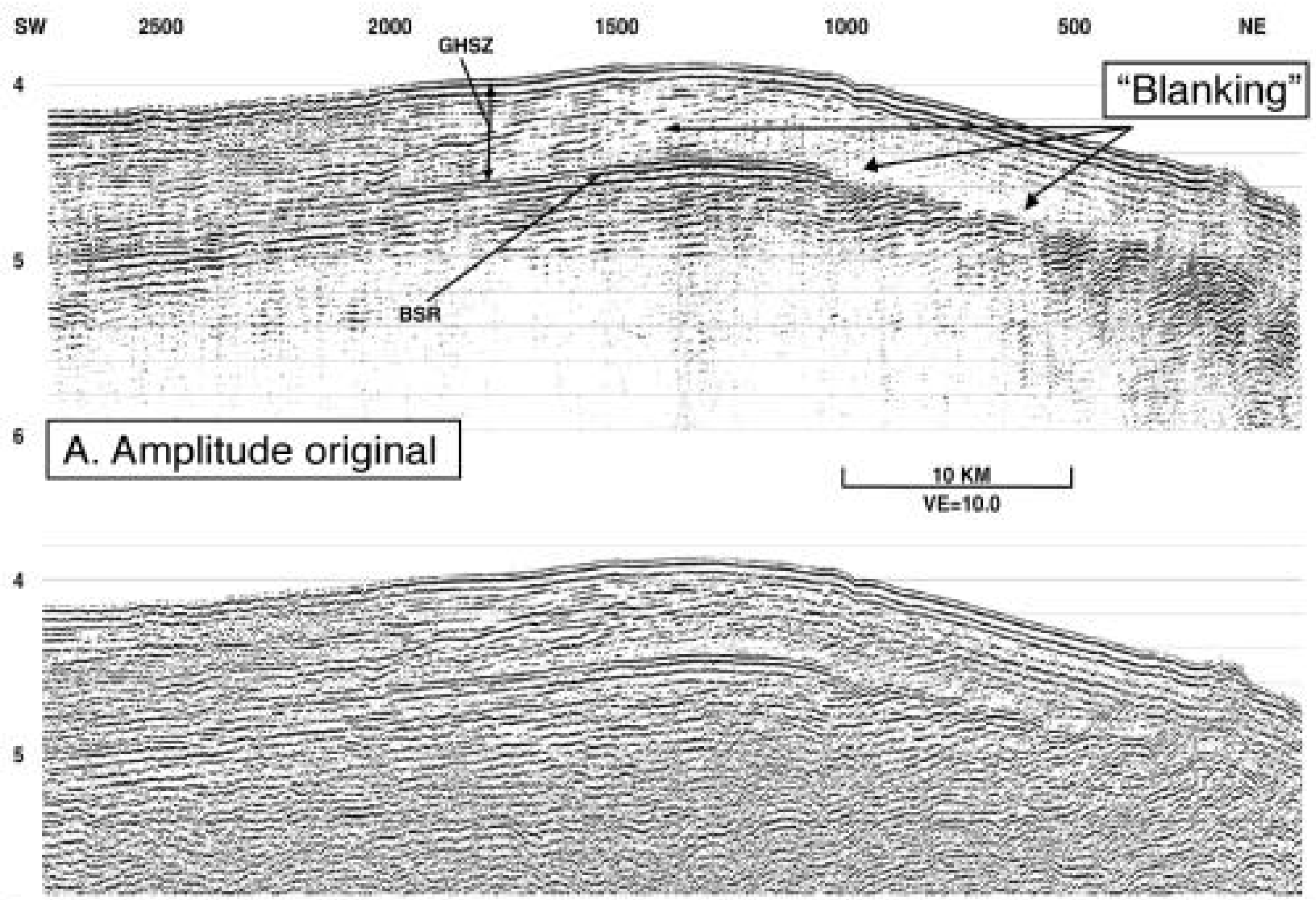

6
B. Amplificação automática do sinal
Hidrato concentrado?

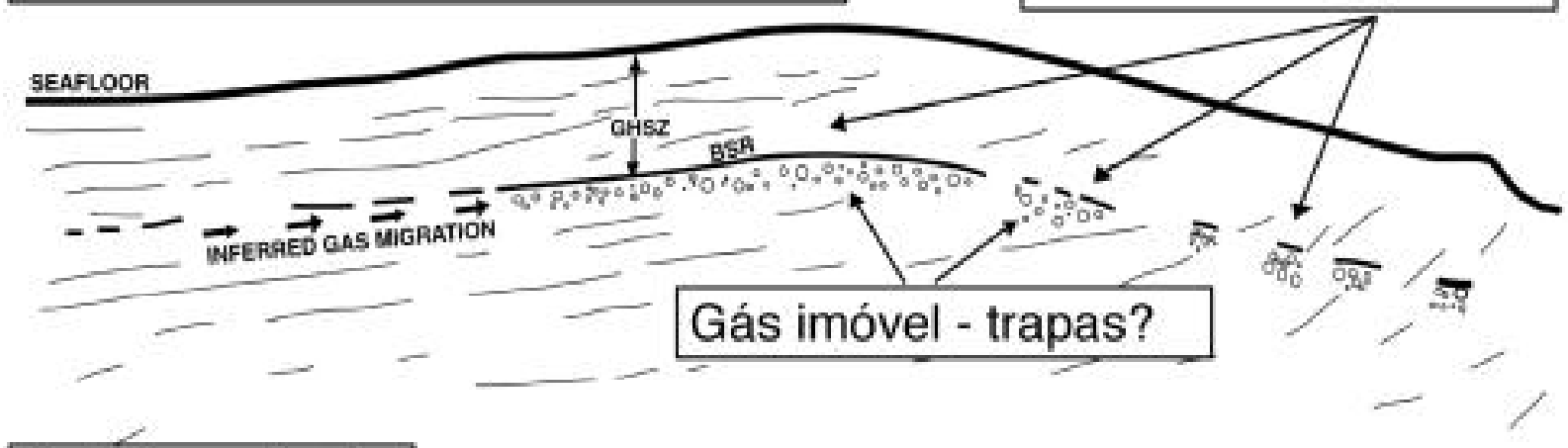

C. Interpretação

Figura 4 - Secção sísmica mostrando um Refletor BSR típico, e o efeito de redução de amplitudes de refletores dentro da zona de estabilidade de hidratos de gás: a chamada "blanking". Localidade: Blake Outer Ridge, na margem passiva do SE dos EUA. Imagem fornecida pelo Dr. William Dillon (USGS); reproduzida de Dillon \& Max (2001) com permissão.

propriedades físicas dos sedimentos, com uma resolução vertical na ordem de centímetros; bem melhor que a resolução sísmica. Estas informações estão necessárias para a verificação das condições no local de onde serão recuperados os testemunhos. Juntos, os dados de perfis e de amostragem dos sedimentos em poços localizados servem como ancoras para a validação dos dados sísmicos adquiridos sobre áreas extensas.

Estudos feitos pelo do Ocean Drilling Program como Leg 164, Blake Ridge (Paull et al., 1996), é o programa nacional japonês conseguiram 
dados de boa qualidade em perfis de poços científicos, incluindo registros de velocidades de ondas $\mathrm{P}$ e $\mathrm{S}$, e eletroresistividade. Os intervalos de sedimentos contendo hidratos de gás mostram, em geral, alta resistividade, elevada $\mathrm{Vp}$, alta $\mathrm{Vs}$, baixa condutividade térmica e baixa constante dielétrica (Goldberg et al., 2000). Com a exceção de intervalos de clatrato maciço, os sinais de raios gama, porosidade e densidade de nêutron, susceptibilidade magnética e efeito fotoelétrico são poucos afetados pela presença de hidratos. Apesar dos sucessos, desenvolvimentos adicionais são necessários para maximizar a informação quantitativa obtidos com as ferramentas de perfilagem convencional (wireline) $\mathrm{e}$ do tipo "Perfilagem Durante a Perfuração" (Logging While Drilling), estas ferramentas sendo desenvolvidas e calibradas pela indústria de petróleo para caracterização de rochas mais duras, menos porosas e mais densas que os sedimentos encontrados na zona de estabilidade de hidratos de gás (0-1000 $\mathrm{m})$.

\section{DETECÇÃO DIRETA E AMOSTRAGEM DE HIDRATOS DE GÁS SUBMARINO}

Normalmente nem testemunhagem rasa nem dragagem não permitem a recuperação de amostras de hidratos de gás. Existem duas explicações. Primeira, a distribuição dos hidratos de gás em sedimentos é muito heterogênea, sendo controlada pela produção de metano biogênico no local, pelos caminhos de transporte pelos gases profundos, e pelo tipo de sedimento. Segunda, a quantidade de metano necessária para estabilizar hidratos está encontrada somente além da profundidade onde todos os íons de sulfato e outras espécies receptoras de elétrons além de $\mathrm{CO}_{2}$ tem sido consumidos. Hidratos de gás encontrados próxima ao fundo marinho normalmente estão associadas à exudações de gás natural ou vulcões de lama (Ginsburg \& Soloviev, 1997). Para obter sucesso na amostragem de hidratos de gás nesses locais, devem ser usadas imagens de alta resolução do fundo marinho e ROV (Remotely Operated Vehicle). Estas tecnologias não vêm sendo amplamente empregadas no Brasil para fins de exploração, contudo essas técnicas são utilizadas em levantamentos geotécnicos em campos em desenvolvimento. Desta forma, a aparente ausência de hidratos de gás na margem continental brasileira pode ser explicada pela pobre esforço de amostragem.

Por outro lado, a ocorrência de hidratos de gás dispersa abaixo da zona de redução de sulfato é apenas acessível através de testemunhos profundos, como as que vem sendo realizadas pelo Ocean Drilling Program (ODP). As perfurações feitas pelo ODP e DSDP têm encontrado hidratos de gás em várias margens passivas e ativas ao redor do mundo. As observações diretas dos cristais de clatrato (Fig. 5) são raras porque o estado sólido é instável nas condições que prevalecem na superfície. As ferramentas capazes de coletar e preservar os sedimentos sob pressão elevada ainda estão sendo desenvolvidas. Normalmente, os hidratos de gás são detectados indiretamente. Por exemplo, uma vez que os hidratos requerem calor para se desassociar, a presença desses em testemunhos pode ser detectada através de medidas de temperatura: as zonas que tenham recém liberado o hidrato ainda registrarão baixas temperaturas (inclusive inferiores a $0^{\circ} \mathrm{C}$ ) (Paull et al., 1996). A forma mais confiável para quantificar os hidratos em testemunhos recuperados parte do fato de que durante a cristalização todos os sais são excluídos da estrutura clatrato; desta forma o mineral consistirá apenas de água pura e gás (Hesse \& Harrison, 1981). Após a cristalização o sal excluído e levado para fora do sistema através de difusão e advecção que ocorre no fluido dos poros (Egeberg \& Dickens, 1999). Quando os testemunhos contendo hidratos são trazidos à superfície os cristais derretem progressivamente, e a água doce liberada dilui o fluido original contido nos poros. Fazendo uma comparação entre a salinidade da água retirado dos poros dos sedimentos com a tendência geral da química da água de acordo com a profundidade é observada a presença de hidratos como uma série de anomalias de água mais doce. Esta tendência é mais bem mostrada na curva de cloro $\left(\mathrm{Cl}^{-}\right)$dissolvido (o cloro não participa em reações diagenéticas).

A magnitude do fluxo vertical do metano é em grande parte responsável pela espessura da zona de redução de sulfato. Com um fluxo maior de metano, o perfil de concentração de sulfato fica menor e mais acentuada, enquanto em áreas com pouca matéria orgânica capaz de gera um fluxo pequeno de metano 


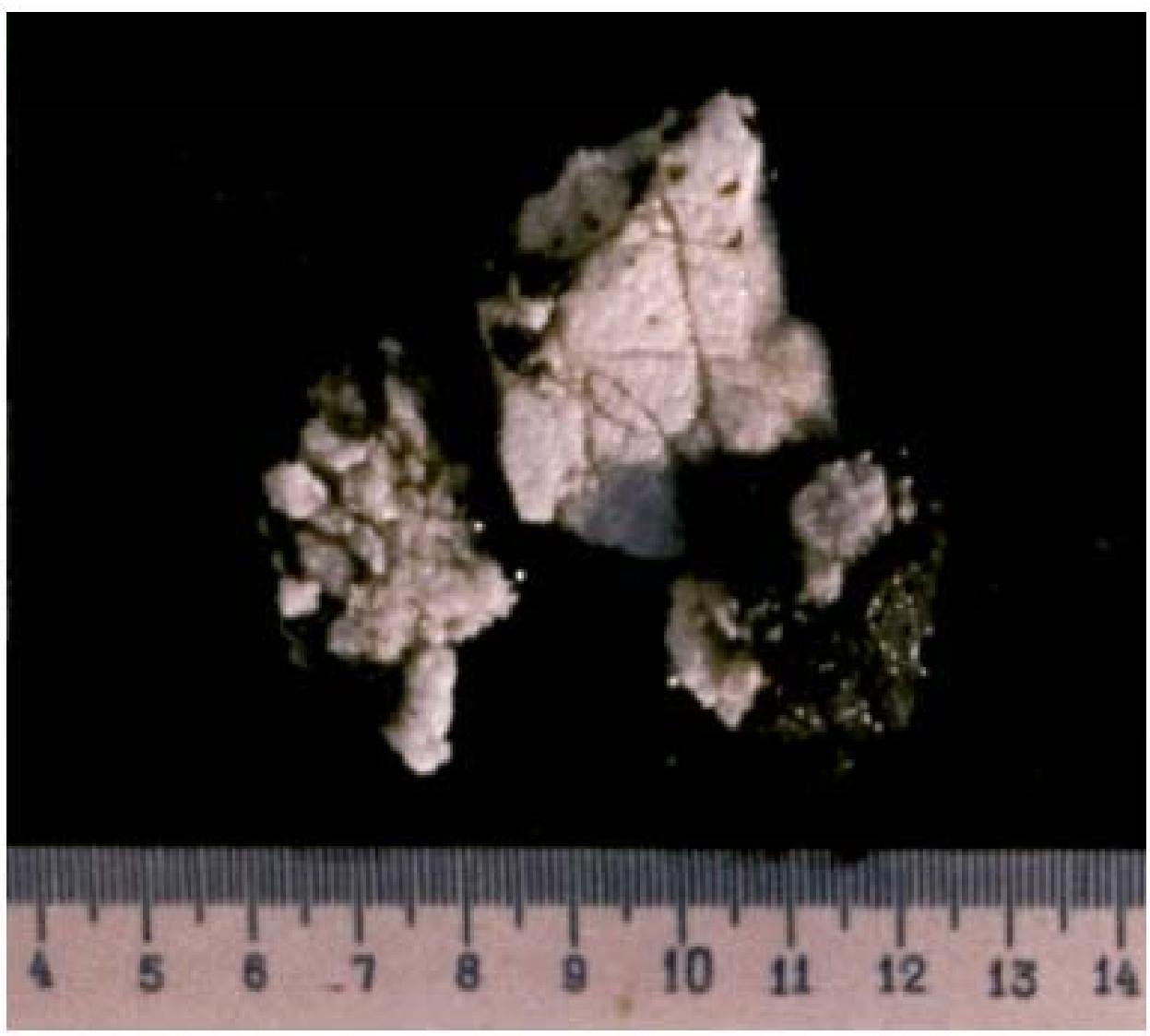

Figura 5 - Cristais de hidrato de gás recuperados de $300 \mathrm{~m}$ embaixo do fundo marinho em sedimentos finos da margem ativa de Costa Rica, durante Leg 170 do Ocean Drilling Program. Imagem fornecida pela Profa. Miriam Kastner (Scripps Institution of Oceanography).

a zona de sulfato pode persistir por dezenas de metros. Um fluxo de metano muito alto implica em concentrações capazes de estabilizar os hidratos de gás a maiores profundidades. Borowski et al. (1999), utilizaram este método de prospecção geoquímica em testemunhos rasas a fim de detectar zonas prováveis de ocorrência de hidratos de gás. O perfilagem do sulfato é um método muito promissor a ser usado para prospecção de hidratos na margem continental brasileira.

As operações convencionais de perfilagem exploratória para óleo e gás não detectam pequenas concentrações de hidratos de gás porque a porção superior do poço é tipicamente perfurada sem recuperação, e depois e feito um revestimento com aço e cimento antes de perfilagem elétrica dessa forma impedindo investigações posteriores. É provável que os hidratos de gás tenham sido perfurados sem detecção, e sem ter apresentado perigo significativo, nas bacias sedimentares brasileiras localizadas em águas profundas. Os hidratos podem ser detectados durante as explorações convencionais para óleo e gás sem comprometimento com a segurança, usando a tecnologia de perfilagem durante a perfuração (Logging While Drilling, LWD), que permite a aquisição de dados petrofísicos na porção mais rasa de um poço exploratório antes deste ser revestido (Boissonnas et al. 2000).

\section{MUDANÇAS CLIMÁTICAS E DINÂMICA DE SISTEMAS MARINHOS DE HIDRATOS DE GÁS}

É necessário compreender como se comportem os hidratos em sedimentos durante mudanças geológicas e ambientais, uma vez que o derretimento dos hidratos pode levar a liberação do metano para os oceanos e atmosfera, onde esse pode contribuir com os pulsos de aquecimento global e escalas decenais ou milenares (Haq, 2000). Por outro 
lado, as mudanças que aumentam a estabilidade dos hidratos podem retirar o carbono do sistema oceanoatmosfera, atuando como um importante sumidouro global em escala geológica (dezenas de milhares a milhões de anos). Um dos cenários envolve uma queda do nível relativo do mar (associada a glaciação), que irá desestabilizar os hidratos, e possivelmente levar a deslizamentos submarinhos que permitam o gás escapar catastroficamente. Este gás contribuiria para o efeito estufa que poderia levar ao término do ciclo glacial, e conseqüentemente à elevação do nível do mar.

As mudanças climáticas passadas tem sido atribuídas às variações no $\mathrm{CO}_{2}$ atmosférico e metano através dos registros quaternários encontrados nos testemunhos de gelo no Ártico e Antártico. Estas correlações têm sido extrapoladas para os períodos em que os climas eram mais extremos que no presente utilizando isótopos de $\mathrm{C}$ e $\mathrm{O}$ em foraminíferos bentônicos e pelágicos. Durante pelo menos um evento de rápido aquecimento no final do Paleoceno, o aumento da assinatura isotópica do carbono leve $\left({ }^{12} \mathrm{C}\right)$ é tão abrupto e intenso que Dickens (1997) interpretou o evento como uma liberação rápida de grandes quantidades de metano a partir de acumulações de hidratos de gás marinho. Na maioria dos casos documentados de mudanças climáticas para o Pleistoceno, o pulso de metano e o carbono leve ocorrem posteriormente aos outros indicadores de aquecimento, isto implica em que as áreas crescentes de tundras onde as geleiras derreteram (altas latitudes), forneceram metano para a atmosfera, e não os hidratos de gás marinho. Todavia, a presença dos hidratos tem sido cada vez mais incluídos nos estudos de modelagem climática para compreender os climas pretéritos e prever mudanças antropogênicas.

\section{DISCUSSÃO}

\section{Hidratos como fonte de combustível?}

Os principais fatores a favor do uso dos hidratos de gás como fontes de energia são:

1. O vasto volume de hidratos submarinhos. Apesar de distribuição dispersa, existem concentrações grandes o suficiente em alguns lugares que justificam a explotação econômica.
2. O crescimento do mercado para o metano (gás natural). $\mathrm{O}$ metano é muito menos poluidor que o óleo e o carvão (não contém enxofre); libera menos dióxido de carbono $\left(\mathrm{CO}_{2}\right)$ para a atmosfera por unidade de energia; e pode ser convertido em combustível líquido (metanol) ou hidrogênio com uso de catalisadores.

3. A presença de hidratos em águas territoriais de países como o Japão e a Índia, cujos países possuem poucos combustíveis fósseis convencionais, garante uma fonte nacional de combustível.

Os principais fatores contra os hidratos como fontes de combustível são:

1. As reservas de óleo e gás ainda são abundantes, e relativamente baratas para a explotação utilizando a infra-estrutura existente.

2. A tendência atual no mercado energético mundial está distante das práticas do uso de combustíveis baseados em carbono, cuja combustão inevitavelmente leva a liberação de $\mathrm{CO}_{2}$.

3. A maioria das acumulações de hidratos de gás existe de forma dispersa, em sedimentos finos e de permeabilidade baixa, inviabilizando a explotação comercial.

4. É necessário gastar energia para derreter o hidrato congelado e liberar o gás. A maioria das perspectivas considera a produção do gás a partir das camadas abaixo da camada de hidratos, provavelmente através de despressurização.

Os programas de pesquisa em hidratos de gás em outros países estão atualmente assumiram duas direções (Bil 2000, Max 2000). Uma delas diz respeito a identificação e quantificação das zonas que contém elevadas concentrações de hidratos de gás que pudesse ser explotado. A outra se refere ao desenvolvimento de tecnologias para a perfuração e produção para fazer uso dessas fontes de combustível. Uma vez que nenhuma combustão de combustível fóssil implica em penalidades em termos de lançamento de $\mathrm{CO}_{2}$ para a atmosfera, os governos têm também liberado verbas para a conversão de metano em metanol ou hidrogênio, ambos podendo ser utilizados em células eletroquímicas altamente eficientes e consideradas tecnologias ultralimpas. Max (2000) enfatiza que o uso de metano em usinas 
termoelétricas convencionais e nestas formas mais limpas (convertidas em hidrogênio e metanol), constituirá uma parte importante no fornecimento de energia no futuro para países desenvolvidos. Contudo, com a importância do Protocolo de Kyoto ainda ignorada por alguns dos países mais poluentes do planeta, esta visão pode parecer de certa forma otimista.

\section{A PERSPECTIVA BRASILEIRA}

Os refletores BSR já foram registrados em estudos geofísicos na margem continental brasileira, na Foz do Amazonas no norte (Sad et al., 1998) até a Bacia de Pelotas no sul (Fontana \& Mussumeci, 1994). Provavelmente, os hidratos de gás ocorrem em muitas outras áreas, desde que haja uma quantidade suficiente de metano biogênico e termogênico. Em bacias sedimentares como a de Campos, Espírito Santo e Cumuruxatiba, onde os diápiros são comuns, é provável que os hidratos de gás ocorram associados a exudações localizadas, como aquelas encontradas no Golfo do México e no Delta do Rio Niger (Hovland et al., 1997).

Assim, poderiam ser sugeridas oportunidades para a explotação comercial de hidratos de gás no Brasil? Considerando a falta de tecnologia disponível e os dados limitados sobre a distribuição dos hidratos de gás no Brasil, ainda é cedo para ser assertivo sobre o futuro desse recurso no país. É possível que em águas profundas exista um tipo de ocorrência de hidrocarbonetos onde os hidratos de gás bloqueiem os poros em arenitos turbidíticos formando assim uma trapa por ação capilar que pudesse permitir a acumulação economicamente viável de gás, ou até mesmo óleo (Grauls et al. 2001; Clennell et al. 2000).

É importante lembrar que para cada geocientista pesquisando a ocorrência natural de hidratos de gás existem dois ou três engenheiros e químicos trabalhando na prevenção dos problemas que podem surgir em linhas de transmissão submarinas e outras instalações. As explorações de petróleo se direcionando a águas profundas e ultraprofundas, a maior limitação econômica para desenvolver muitos campos pequenos e remotos é o grande custo envolvido na instalação e manutenção de equipamentos para injetar e retirar produtos químicos inibidores da formação de hidratos de gás. Mesmo que as concentrações economicamente viáveis de hidratos, e os meios de explotá-las nunca forem desenvolvidos, o impacto econômico destes compostos ao setor de óleo e gás no Brasil será ainda imenso.

\section{CONCLUSÕES E RECOMENDAÇÕES}

A maior motivação para estudar hidratos de gás é que esses compostos formam um elemento chave no ciclo global do carbono, o qual está envolvido em fortes feedbacks e eventos catastróficos. Apesar a falta de evidência concreta, ainda está provável que ao longo dos $8000 \mathrm{~km}$ da margem continental brasileira se encontram várias ocorrências de hidratos de gás de importância mundial.

Para compreender os hidratos de gás marinhos, é necessário levar em consideração a termodinâmica, o metabolismo e a estrutura das comunidades microbióticas, o ciclo do carbono, os fluxos de difusivos e advectivos de calor, água e metano na subsuperfície e os processos geológicos em escala de tempo milenar. Já para detectar os clatratos, é necessário utilizar uma combinação de métodos geofísicos, petrofísicos e geoquímicos, incluindo análises de biomarcadores e isótopos. Mas para amostrar os hidratos, é necessário lançar mão de técnicas como testemunhagem profunda ou testemunhagem rasa localizada através de ROV. Assim, um esforço multidisciplinar é necessário, requerendo uma mobilização de recursos consideráveis para realizar uma exploração de forma quantitativa.

Com este conhecimento prévio, a perspectiva de recuperação econômica dos hidratos de gás é muito remota. Mesmo assim, os clatratos podem oferecer retorno excepcional no caso da ocorrência em grande concentração em sedimentos permeáveis, dos quais o gás pudesse ser extraído. No cenário exploratório brasileiro, qualquer descoberta dessa natureza seria fortuita, e um produto secundário das atividades exploratórias normais. É difícil fazer uma previsão para um programa nacional de recursos estratégicos de hidratos na forma adotada por países como Japão, E.U.A., Índia e na U.E., mas 
cooperações técnicas e científicas com esses países poderiam ser vantajosas.

\section{AGRADECIMENTOS}

$\mathrm{MBC}$, atualmente bolsista - Pesquisador Visitante, reconhece o apoio do CNPq. Martin Hovland (Statoil) e Bill Dillon (United States Geological Survey) forneceram linhas sísmicas. Bahman Tohidi (Heriot Watt University) facilitou inúmeras colaborações.

\section{REFERÊNCIAS}

ANDREASSEN, K., HART, P.E. \& MACKAY, M., 1997. Amplitude versus offset modeling of the bottom simulating reflecton associated with submarine gas hydrates, Mar. Geol., 137: 25-40.

BIL, K.J., 2000. Economic perspective of methane from hydrate. In: Natural gas Hydrate in Oceanic and Permafrost Environments (Max. M.D., ed.), Kluwer, Dordrecht, 349-360.

BOISSONNAS, R., GOLDBERG, D. \& SAITO, S., 2000. Electromagnetic modelling and in situ measurement of gas hydrate in natural marine environments. Ann. N.Y. Acad. Sci., 912: 159166.

BOROWSKI, W.S., PAULL, C.K. \& USSLER, III, W., 1999. Global and local variations of interstitial sulfate gradients in deep-water, continental margin sediments: Sensitivity to underlying methane and gas hydrates. Mar. Geol., 159: 131-154.

BUFFETT, B.A., 2000. Natural Gas Hydrates. Ann. Rev. Earth. Plan. Sci., 20: 1567-1570.

CARPENTER, G. - 1981 Coincident slumping/gas hydrate occurrences on the US Atlantic Continental slope. Geo-Mar. Lett., 1: 29-32.

CLENNELL, M.B., JUDD, A.G. \& HOVLAND, M., 2000. Movement and accumulation of methane in marine sediments: relation to gas hydrate systems. In: Natural gas Hydrate in Oceanic and Permafrost Environments (Max. M.D., ed.), Kluwer, Dordrecht, 105-122.

DAVIE, M.K. \& BUFFETT, B.A., 2001. A numerical model for the formation of gas hydrate below the sea floor. J. Geophys. Res., 106: 497514.
DICKENS, G.R., CASTILLO, M.M., \& Walker, J.C.G., 1997. Alast of gas in the latest Palaeocene: similating first-order effects of massive dissociation of oceanic methane hydrate. Geology, 25: 259-262.

DVORKIN, J., HELGERUD, M.B., WAITE, W.F., KIRBY, S.H. \& NUR, A., 2000. Introduction to physical properties and elasticity models. In: Natural gas Hydrate in Oceanic and Permafrost Environments (Max. M.D., ed.), Kluwer, Dordrecht, 245-260.

ECKER, C., Dvorkin, J. \& Nur, A., 2000. Estimating the amount of hydrate and free gas from surface seismic. Geophysics, 65: 565-573.

EDWARDS, R.N., 1997. On the resource evaluation of marine gas hydrate deposits using sea-floor transient electric dipole methods. Geophysics 62 , 63-74.

EGEBERG, P.K. \& DICKENS, G.R., 1999. Thermodynamic and pore water halogen constraints on gas hydrate distribution at ODP Site 997 (Blake Ridge), Chem. Geol., 153: 53-79.

FISHER C.R., MACDONALD I.R., SASSEN R., YOUNG C.M., MACKO S.A., HOURDEZ S., CARNEY R.S., JOYE S. \& MCMULLIN, E., 2000. Methane ice worms: Hesiocaeca methanicola colonizing fossil fuel reserves. Naturwiss., 87: 184-187.

FONTANA, R.L., \& MUSSUMECI, A., 1994. Hydrates offshore Brazil, Ann. N.Y. Acad. Sci, 715: 106-113.

GINSBURG, G.D. \& SOLOVIEV, V., 1997. Methane migration within the submarine gashydrate stability zone under deep-water conditions: Mar. Geol., 137: 49-57.

GOLDBERG，D.S., COLLETT, T.S. \& HYNDMAN, R.D., 2000. Ground trusth: in-situ properties of hydrate. In: Natural gas Hydrate in Oceanic and Permafrost Environments (Max. M.D., ed.), Kluwer, Dordrecht, 295-309.

GRAULS, D., 2001. Gas hydrates: importance and applications in petroleum exploration. Mar Petrol Geol 18: 519-523.

HAQ, B.U., 2000. Climate impact of nature gas hydrate. In: Natural gas Hydrate in Oceanic and Permafrost Environments (Max. M.D., ed.), Kluwer, Dordrecht, 137-148. 
HARVEY, L.D.D. \& HUANG, Z., 1995. Evaluation of the potential impact of methane clathrate destabilization on future global warming: J. of Geophys. Res., D100: 2905-2926.

HESSE, R., \& HARRISON, W.E., 1981. Gas hydrates (clathrates) causing pore-water freshening and oxygen isotope fractionation in deep water sedimentary sections of terrigenous continental margins, Earth Planet. Sci. Lett., 55: 603-613

HOBRO, J.W., MINSHULL, T.A. \& SINGH, S.C., 1998. Tomographic studies of the methane hydrate stability zone in the Cascadia Margin. In: Gas Hydrates: Relevance to World Margin Stability and Climate Change (Henriet, J.-P. \& Mienert, J., eds.) Geol. Soc. Lond. Spec. Publ. 137: 133-140.

HOLBROOK, W.S., HOSKINS, H., WOOD, W.T., STEPHEN, R.A., LIZARRALDE, D. \& LEG 164 SCIENTIFIC PARTY, 1996. Methane hydrate and free gas on the Blake Ridge from vertical seismic profiling: Science, v. 273, p. 1840-1843.

HOVLAND, M., GALLAGHER, J.W., CLENNELL, M.B. \& LEKVAM, K., 1997. Gas hydrates and free gas volumes in marine sediments: Example from the Niger Delta front. Mar. \& Pet. Geol., 14: 245-255.

KVENVOLDEN, K.A., 1993. Gas hydratesgeological perspective and global change: Rev. Geophys., v. 31, p. 173-187.

LEE, M.W., HUTCHINSON, D.R., DILLON, W.P., MILLER, J.J., AGENA, W.F. \& SWIFT, B.A., 1993. Method of estimating the amount of in-situ gas hydrates in deep marine sediments. Mar. Pet. Geol., 10: 493-506.

MACKAY, M.E., JARRARD, R.D., WESTBROOK, G.K., HYNDMAN, R.D. \& SHIPBOARD SCIENTIFIC PARTY OF ODP LEG 146, 1994. Origin of bottomsimulating reflectors: Geophysical evidence from the Cascadia accretionary prism: Geology, v. 22, p. 459-462.

MILES, P.R., 2000. Geophysical sensing and hydrate. In: Natural gas Hydrate in Oceanic and Permafrost Environments (Max. M.D., ed.), Kluwer, Dordrecht, 261-274.
MINSHULL, T.A., SINGH, S.C. \& WESTBROOK, G.K., 1994. Seismic velocity structure at a gas hydrate reflector, offshore western Colombia, from full waveform inversion. J. Geophys. Res., 99: 4715-4734.

PAULL, C.K., MATSUMOTO, R., WALLACE, P. AND LEG 164 SCIENTIFIC PARTY, 1996. Proceedings, ODP Initial Reports, v. 164, College Station, TX, (Ocean Drilling Program).

PAULL, C.K., USSLER III, W. \& DILLON, W.P., 2000. Potential role of gas hydrate in generating submarine slope failures. In: Natural gas Hydrate in Oceanic and Permafrost Environments (Max. M.D., ed.), Kluwer, Dordrecht, 149-155.

PAULL, C.K., USSLER III, W. AND BOROWSKI, W.S., 1994. Sources of methane to form marine gas hydrates, Ann. N.Y. Acad. Sci, 715: 392-409.

PECHER, I. \& HOLBROOK, W.S., 2000. Seismic methods for detecting and quantifying gas hydrates. In: Natural gas Hydrate in Oceanic and Permafrost Environments (Max. M.D., ed.), Kluwer, Dordrecht, 275-294.

SAD, A.R.E, SILVEIRA, D.P., MACHADO, D.A.P., SILVA, S.R.P. \& MACIEL, R.R., 1998. Marine gas hydrates evidence along the Brazilian coast. Proc. AAPG international conference and exhibition. Rio de Janeiro, Brazil. Nov. 8-11, (CD-Rom).

SHIPLEY, T.H., HOUSTON, M.H., BUFFLER, R.T., SHAUB, F.J., McMILLEN, K.J., LADD. J.W AND WORZEL, J.L., 1979. Seismic evidence for widespread possible gas hydrate horizons on continental slopes and rises: Am. Assoc. Petrol. Geol. Bull., 63: 22042213.DILLON, W.P. \& MAX, M.D. - 2001 Gas hydrate in seafloor sediments: impact on future resources and drilling safety. Offshore Technology Conference 2001, Proceedings, CD ROM, OTC \#13034, 10 pp.

SLOAN, E.D., 1998. Clathrate hydrates of natural gas: $2^{\text {nd }}$ edition. Marcel Dekker, New York. 436 pp.

TINIVELLA， U., LODOLO, E., CAMERLENGHI, A. \& BOEHM, G., 1998. Seismic tomography study of a bottom simulating 
reflector off the South Shetland Islands (Antarctica). In: Gas Hydrates: Relevance to World Margin Stability and Climate Change (Henriet, J.-P. \& Mienert, J., eds.) Geol. Soc. Lond. Spec. Publ. 137: 141-151.

WELLSBURY, P. \& PARKES, R.J., 2000. Deep biosphere: source of methane for oceanic hydrate. In: Natural gas Hydrate in Oceanic and Permafrost Environments (Max. M.D., ed.), Kluwer, Dordrecht, 91-104.
WILLOUGHBY, E.C., LATYCHEV, K., EDWARDS, R.N. \& MIHAJLOVIC, G., 2000. Resource evaluation using seafloor compliance methods. Ann. N.Y. Acad. Sci. 912, 146-158.

WOOD, W.T., STOFFA, P.L. \& SHIPLEY, T.H., 1994. Quantitative detection of methane hydrate through high-resolution seismic velocity analysis. J. Geophys. Res., 99: 9681-9695.

\section{NOTE ABOUT THE AUTHOR}

\section{MICHAEL BENEDICT CLENNELL}

Professor Visitante no Centro de Pesquisa em Geofísica e Geologia da Universidade Federal da Bahia desde 1999. Atual bolsista de Pesquisador Visitante do CNPq, e Cientista Visitante no Centro de Excelência em Hidratos de Gás na Universidade de Heriot Watt (Escócia).

Formação acadêmica: Graduação em Geologia na Universidade de Oxford, Inglaterra, 1988. Doutorado em Geologia na Universidade de Londres, 1992.

Histórico Professional: Pesquisa no nível de pósdoutorado entre 1992 e 1999. Na Universidade de Birmingham 1992-1993 trabalhou na área de geofísica marinha. Na Universidade de Leeds, 1993-1999 trabalhou com vários grupos internacionais nas áreas de geologia marinha, geologia do petróleo, petrofísica e hidratos de gás. Participou em 2 pernadas do Ocean Drilling Program e ganhou o título "Rob Kidd ODP Fellow" do Natural Environment Research Council 1997-1999. Atuou como pesquisador visitante no projeto de Hidratos de Gás no United States Geological Survey, Marine Division, Woods Hole, 1995-1999. Sócio de várias sociedades profissionais: Geological Society of London, Sociedade Brasileira de Geofísica, American Geophysical Union, Society of Petroleum Engineers, Sociedade Brasileira de Geologia de Petróleo. Áreas de Interesse: Geologia do Petróleo, Petrofísica Aplicada à Hidrogeologia, Hidratos de Gás Submarinhos, Geologia Estrutural (deformação de sedimentos e rochas).

\section{UNESP - University of the State of São Paulo}

\section{IGCE - Institute of Geosciences and Exact Sciences}

The IGCE-UNESP provides training on geophysical methods at undergraduate and graduate levels, where the main areas of interest are Engineering Geology, Hydrogeology, Environmental Geology, Geotectonics, and Nuclear Geophysics. The training is principally supplied to students of the undergraduate course in Geology, as well to professionals attending the graduate courses in Geosciences, whose subject areas are Geosciences and Environment and Regional Geology. Browse http://www.re.unesp.br 


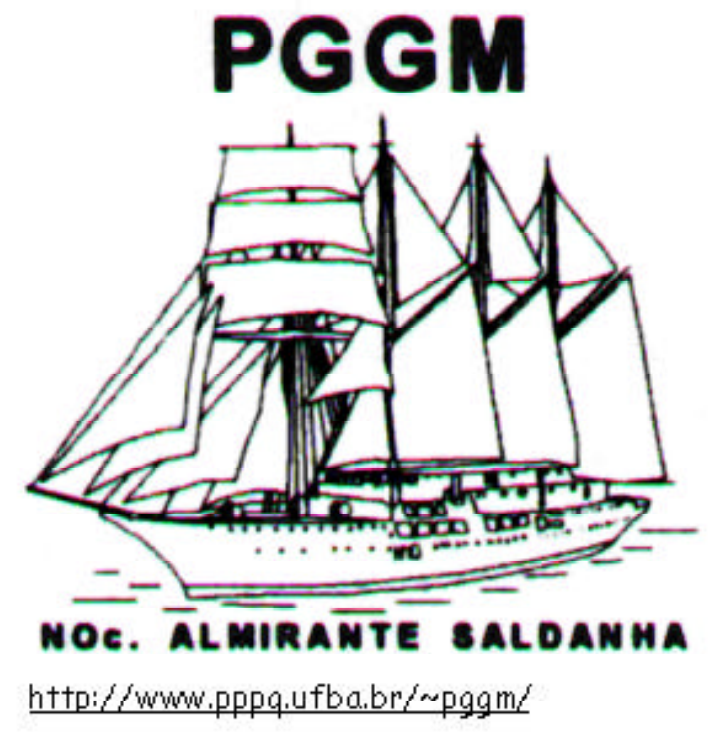

Instituições efetivas

Laboratório de OceanografiarUPA LABOHIDRO,Univ. Federal do Maranhäo - UFMA LGMa/Univ. Federal do Ceará - UFC Departamento de Geociâncias/UECE DOL/Univ. Federal do Rio Grande do Norte - UFRN NEPREMaR / Univ. Federal da Paraíba - UPB LGGWUniv. Federal de Permambuco - UFPE DEPESCA /Univ. Federal Raral de Pemambuco - URRE PPPG/Lniv. Federal da Bahia - UFA

Lab. de Geomorfologia Fluvial Costeira e Submaria/UFRJ Laboratório de Geologia Marinha - LAGEMAR/UFF Departamento de Oceanografia/UERT Instituto Oecanogrúfico - IO/USP

FACIMAR/Univ. do Vale do Itajaí - UNIVALI Departamento de Geociêncio - GCN/UFSC CECO/Univ. Federal do Pio Gronde do Sul - UFPGS Laboratório de Oceanografía Geológica'FURG

\section{Instituições colaboradoras}

Miniztério da Ciêneia e Tecnologia - MCT

Miniztério do Meio Ambiente, dos Recurzos Hídricos e da Amazônia Legal - MWA

Conselho Nacional de Desenvolvimento Científico e Tecnalógico - CNPq

Comisão Interminieterial para $\propto$ Recursos do Mar - CIRM

Companhia de Pexpiza de Recursos Mineraiz - CPRM

Departamento Nacional da Produgäo Mineral - DNPM

Diretoria de Hidrografia e Navegagão - DHN/MM

Fundagão dos Etudos do Mar - FEMAR

Petróleo Breileiro S/A - PETROBRAS
- Programa de Geologia e Geofísica Marinha foi constituído em 1969 através do Conselho Nacional de Desenvolvimento Científico e Tecnológico ( $\mathrm{CNPq}$ ), da Diretoria de Hidrografia e Navegação (DHN) da Marinha do Brasil, do Departamento Nacional da Produção Mineral (DNPM) e das Universidades brasileiras. $O$ programa tem como objetivo realizar levantamentos geológicos e geofísicos sistemáticos na Margem Continental Brasileira, destacando-se o seguinte:

- Realizar estudos na zona costeira. margem continental $e$ fundo oceânico. visando elaborar mapeamentos, avaliar o potencial de recursos minerais, subsidiar os programas de delimitação da plataforma continental e fornecer dados que possam servir para o aprimoramento de estudos integrados com áreas a fins:

- Formar e capacitar pessoal em geologia marinha e geofísica marinha:

- Fortalecer centros de excelência de pesquisa e ensino existentes no Brasil e apoiar grupos emergentes participantes do Programa.

Coordenação do Programa

UFS C - Departamento de Geociências - GCN

Prof. Norberto Olmiro Hom Filho

Depto. Geociências-CFH-UFSC

Campus Universitário - Trindade Cx.Postal 476

Florianópolis - SC

$88040-900$

Fone: (48) 3319286

Fax: (48) 3319751

E-mail: hom(dicth.ufsc.br 